\title{
Evidence-based recommendations on storing and handling specimens for analyses of insect microbiota
}

Tobin J Hammer, Jacob C Dickerson, Noah Fierer

Research on insect microbiota has greatly expanded over the past decade, along with a growing appreciation of the microbial contributions to insect ecology and evolution. Many of these studies use DNA sequencing to characterize the diversity and composition of insect-associated microbial communities. The choice of strategies used for specimen collection, storage, and handling could introduce biases in molecular assessments of insect microbiota, but such potential influences have not been systematically evaluated. Likewise, although it is common practice to surface sterilize insects prior to DNA extraction, it is not known if this time-consuming step has any effect on microbial community analyses. To resolve these methodological unknowns, we conducted an experiment wherein replicate individual insects of four species were stored intact for two months using five different methods-freezing, ethanol, dimethyl sulfoxide (DMSO), cetrimonium bromide (CTAB), and room-temperature storage without preservative-and then subjected to whole-specimen 16S rRNA gene sequencing to assess whether the structure of the insect-associated bacterial communities was impacted by these different storage strategies. Overall, different insect species harbored markedly distinct bacterial communities, a pattern that was highly robust to the method used to store samples. Storage method had little to no effect on assessments of microbiota composition, and the magnitude of the effect differed among the insect species examined. No single method emerged as "best," i.e. one consistently having the highest similarity in community structure to control specimens, which were not stored prior to homogenization and DNA sequencing. We also found that surface sterilization did not change bacterial community structure as compared to unsterilized insects, presumably due to the vastly greater microbial biomass inside the insect body relative to its surface. We therefore recommend that researchers can use any of the methods tested here, and base their choice according to practical considerations such as prior use, cost, and availability in the field, although we still advise that all samples within a study be handled in an identical manner when possible. We also suggest that, in large-scale molecular studies of hundreds of insect specimens, surface sterilization may not be worth the time and effort involved. This information should help researchers design sampling strategies and will facilitate cross- 
comparisons and meta-analyses of microbial community data obtained from insect specimens preserved in different ways. 
1 Evidence-based recommendations on storing and handling specimens for analyses of insect

2 microbiota

3

4 Tobin J. Hammer ${ }^{1,2}$, Jacob C. Dickerson ${ }^{1}$, Noah Fierer ${ }^{1,2}$

5 1. Department of Ecology and Evolutionary Biology, University of Colorado at Boulder,

6 Boulder, CO, USA

7 2. Cooperative Institute for Research in Environmental Sciences, University of Colorado at

8 Boulder, Boulder, CO, USA

9

10 Corresponding author:

11 Tobin J. Hammer

12 University of Colorado at Boulder, CIRES Bldg. Rm. 318, Boulder, CO, 80309-0001

$131(650) 996-1315$

14 tobin.hammer@colorado.edu

15 


\section{Abstract}

Research on insect microbiota has greatly expanded over the past decade, along with a growing appreciation of the microbial contributions to insect ecology and evolution. Many of these studies use DNA sequencing to characterize the diversity and composition of insectassociated microbial communities. The choice of strategies used for specimen collection, storage, and handling could introduce biases in molecular assessments of insect microbiota, but such potential influences have not been systematically evaluated. Likewise, although it is common practice to surface sterilize insects prior to DNA extraction, it is not known if this timeconsuming step has any effect on microbial community analyses. To resolve these methodological unknowns, we conducted an experiment wherein replicate individual insects of four species were stored intact for two months using five different methods - freezing, ethanol, dimethyl sulfoxide (DMSO), cetrimonium bromide (CTAB), and room-temperature storage without preservative - and then subjected to whole-specimen 16S rRNA gene sequencing to assess whether the structure of the insect-associated bacterial communities was impacted by these different storage strategies. Overall, different insect species harbored markedly distinct bacterial communities, a pattern that was highly robust to the method used to store samples. Storage method had little to no effect on assessments of microbiota composition, and the magnitude of the effect differed among the insect species examined. No single method emerged as "best," i.e. one consistently having the highest similarity in community structure to control specimens, which were not stored prior to homogenization and DNA sequencing. We also found

37 that surface sterilization did not change bacterial community structure as compared to

38 unsterilized insects, presumably due to the vastly greater microbial biomass inside the insect 
39 body relative to its surface. We therefore recommend that researchers can use any of the methods

40 tested here, and base their choice according to practical considerations such as prior use, cost,

41 and availability in the field, although we still advise that all samples within a study be handled in

42 an identical manner when possible. We also suggest that, in large-scale molecular studies of

43 hundreds of insect specimens, surface sterilization may not be worth the time and effort

44 involved. This information should help researchers design sampling strategies and will facilitate

45 cross-comparisons and meta-analyses of microbial community data obtained from insect

46 specimens preserved in different ways.

Introduction

Many insects are associated with microbial symbionts that play critical roles in their

51 ecological interactions and have shaped their evolutionary history (Dillon \& Dillon, 2004; Engel

52 \& Moran, 2013; Douglas, 2015). The structure and function of insect microbiota are increasingly

53 studied using molecular methods such as 16S rRNA gene sequencing, but these methods are

54 often directly adopted from environmental or human microbiota studies and have rarely been evaluated for their efficacy and accuracy when used with insect specimens. The growing field of

56 insect-microbial symbiosis would benefit from improved validation of the sample storage and

57 handling strategies used for studying insect microbiota.

58 Sample storage is one of the most important steps to consider when designing and

59 implementing any DNA sequencing-based analysis of insect-associated microbial communities.

60 When insect specimens are collected in the field, it is often not possible to extract DNA

61 immediately after insect collection, and so specimens must be preserved in some way that 
62 minimizes the impact to microbial community structure. Samples are often irreplaceable, and if

63 they are preserved improperly then they will remain useless no matter what technological

64 advances in DNA extraction, amplification, or sequencing arise in the future (Cary \& Fierer, 65 2014).

66 While some studies have shown that storage methodology can affect DNA recovery and 67 amplification success from insects and/or their symbionts (Post, Flook \& Millest, 1993; Fukatsu, 68 1999; Mandrioli, Borsatti \& Mola, 2006; Moreau et al., 2013), we know of no study that has 69 directly tested how different storage methods impact molecular assessments of insect-associated 70 microbial community structure. Previous studies of insect microbiota have used a range of 71 storage methods including, but not limited to, freezing (Jones, Sanchez \& Fierer, 2013; Hammer, 72 McMillan \& Fierer, 2014), ethanol (Koch et al., 2013; Estes et al., 2013), RNAlater (Campbell et 73 al., 2004; Sanders et al., 2014), and combinations thereof. We do not know how these different 74 storage strategies may influence assessments of microbial community structure and whether 75 some storage strategies are better suited for some insect groups than others. Furthermore, it 76 remains poorly understood whether and to what degree any potential biases associated with 77 sampling handling strategies may influence conclusions from meta-analyses (Colman, Toolson 78 \& Takacs-Vesbach, 2012) or individual studies surveying microbiota of insects stored in 79 different ways.

80 Storage methods could impact insect microbiota through differential preservation of some 81 microbial cell types (e.g., gram-positive versus gram-negative bacteria), differential penetration 82 into insect tissues, or due to the preservative itself contaminating the specimen. Studies 83 comparing the most commonly used storage methods on non-insect samples (e.g., soil, plant 84 leaves, and human feces) have generally found that effects on assessments of bacterial 
85 community structure are nonexistent, or negligible in magnitude relative to biological factors that

86 are of interest such as interindividual or interspecific differences in microbiota (Lauber et al.,

87 2010; Rubin et al., 2013; U’Ren et al., 2014; Dominianni et al., 2014; Franzosa et al., 2014).

88 A distinct, but related issue in processing insect specimens for molecular analysis of their

89 microbiota is determining whether the specimens require surface sterilization prior to DNA

90 extraction. This approach typically involves soaking specimens in dilute bleach and is often

91 employed to remove any surface-associated microbes or contaminants from handling or storage

92 reagents so that downstream analyses will just capture internal symbionts, such as those in the

93 gut, bacteriomes, or reproductive structures. Despite the widespread use of surface sterilization

94 (e.g., Rosengaus et al., 2011; Hammer, McMillan \& Fierer, 2014; Sanders et al., 2014) and the

95 significant amount of time it adds when extracting DNA from hundreds of specimens, its

96 potential impact on molecular assessments of insect microbiota has not yet been evaluated. If the

97 vast majority of microbial biomass is found inside insects, surface sterilization might have little

98 effect on the whole-insect microbiota. Alternatively, if the procedure is too aggressive, surface

99 sterilization could decrease the abundance of internal microbiota and introduce biases in the

100 assessment of microbial community composition.

101 In this study, we asked: i) Do storage and surface sterilization methods affect insect-

102 associated microbial community structure, and if so, are these effects dependent on the insect

103 species examined?, and ii) How do potential methodological biases compare in magnitude

104 relative to the (presumably large) inter-order differences in microbiota? To address these

105 questions, we conducted an experiment on replicate individuals from four morphologically and

106 phylogenetically disparate insect species collected from the same locality. Specimens were

107 stored for two months using five different treatments before DNA extraction for 16S rRNA- 
108 based community profiling. Our goal was to develop and validate a set of "best practices" for the

109 storage and handling of samples prior to DNA-based investigations of insect microbiota.

110

111 Materials and Methods

112

113 During the summer of 2013, we collected adult specimens of four insect species in

114 Boulder, Colorado, USA: the cabbage white butterfly Pieris rapae (Lepidoptera: Pieridae), the

115 speckle-winged grasshopper Arphia conspersa (Orthoptera: Acrididae), the Mexican bean beetle

116 Epilachna varivestis (Coleoptera: Coccinellidae), and the honey bee Apis mellifera

117 (Hymenoptera: Apidae). We chose to collect the specimens as they would typically be collected

118 by entomologists in order to assess the potential value of previously collected insect specimens

119 for molecular microbial studies, and to evaluate the role of surface sterilization in removing

120 potential contaminants introduced from human skin or collection equipment when samples are

121 not collected or handled aseptically. For example, we used standard nets as well as manual

122 collection (without gloves), and sacrificed insects in jars containing ethyl acetate (Willows-

123 Munro \& Schoeman, 2014). Insect bodies were stored intact, except butterflies from which we

124 first removed the wings.

125 By collecting multiple members of the same population at the same time, we aimed to

126 minimize external sources of microbiota variability and thus maximize the potential to detect

127 storage treatment effects. Unlike previous studies on soil or feces (e.g., Lauber et al., 2010;

128 Rubin et al., 2013), a single insect sample cannot be equally divided into sub-samples to test

129 multiple storage treatments; hence, our replicates are individual specimens and we expected

130 some natural variability in microbiota among them. Six individuals were collected for each 
131 species-treatment combination, although not all specimens yielded sufficient sequence data for

132 inclusion in downstream analyses. An overview of our experimental design is shown in Figure 1.

133 To test for surface sterilization effects, one set of specimens (chosen randomly from those

134 collected) were surface-sterilized immediately after field collection through a rinse in sterile

135 water (Sigma-Aldrich), a soak in 70\% ethanol followed by $10 \%$ bleach for $60 \mathrm{~s}$ each, and a

136 second rinse in water (similar to Hammer et al., 2014, though without subsequent immersion in

137 liquid $\mathrm{N}_{2}$ ). We then thoroughly homogenized the insects with a sterile mortar and pestle, in order

138 to recover DNA from all potential internal symbionts (Rubin et al., 2014). The unsterilized

139 control specimens were homogenized directly after field collection. All homogenized material

140 was frozen at $-80^{\circ} \mathrm{C}$ until DNA extraction.

141 To test for effects of different storage strategies, whole insects were stored (without prior

142 surface sterilization) over eight weeks using one of five different methods detailed below, and

143 then homogenized and frozen at $-80^{\circ} \mathrm{C}$. These samples were later compared to the controls

144 (specimens homogenized directly after field collection and frozen at $-80^{\circ} \mathrm{C}$ prior to DNA

145 extraction). Although it was not possible to extract microbial DNA from freshly caught insects,

146 we expected the controls to be the most similar to wild insect microbiota, and thus consider them

147 to have the fewest potential biases of any of our treatments. One storage treatment simply

148 involved maintaining dead insects in empty tubes in the laboratory, under ambient conditions

149 (approximately $21^{\circ} \mathrm{C}$ and $32 \%$ humidity). This treatment mimics how insects are often stored in

150 museum collections, and we expected this treatment to have the strongest effect relative to the

151 control.

152 The second storage method involved freezing specimens dry (i.e., no preservative) at -

$15320^{\circ} \mathrm{C}$. Freezing is commonly used and should have a lower potential for introducing 
154 contaminants than using reagents, but is often impractical in the field and makes sample

155 transport difficult. The final three storage methods - $95 \%$ ethanol, dimethyl sulfoxide (DMSO)

156 and cetrimonium bromide (CTAB) — are all liquid preservatives that can be used without a

157 freezer. Storage in ethanol is frequently used in insect microbiota studies, and ethanol is

158 relatively inexpensive and easy to acquire in the field, but its flammability often makes it

159 unsuitable for transporting specimens on planes (Moreau et al., 2013). DMSO and CTAB are

160 promising nonflammable chemicals that have also been used to preserve specimens for later

161 DNA sequencing (Nagy, 2010). DMSO and CTAB were salt-saturated and prepared at $20 \%$ and

$1622 \%$ concentrations, respectively. We note that these storage methods are sometimes used in

163 combination - e.g., ethanol storage at $-20^{\circ} \mathrm{C}-$ but here we evaluate them separately.

164 We extracted total DNA from subsamples of whole insect homogenates-which were

165 further pulverized with an aggressive bead-beating step —using the MoBio Powersoil Kit, from

166 which a $\approx 300$ bp portion (V4 region) of the bacterial $16 \mathrm{~S}$ rRNA gene was then PCR-amplified,

167 as previously described (Barberán et al., 2014). The barcoded amplicons were pooled and

168 sequenced on the Illumina MiSeq platform (Caporaso et al., 2012). Sequence data were quality-

169 filtered and clustered into operational taxonomic units (OTUs) at the $97 \%$ level using the

170 UPARSE pipeline (Edgar, 2013) following (Ramirez et al., 2014). OTU taxonomic affiliations

171 were assigned using the RDP classifier (Wang et al., 2007) against the August 2013 version of

172 the Greengenes database (McDonald et al., 2012), and OTUs identified as mitochondria or

173 chloroplasts were removed. The total proportion of mitochondrial or chloroplast reads for each

174 insect species was: bees, 0.02 ; grasshoppers, 0.38 ; beetles, 0.20 ; butterflies, 0.12 . Finally, to

175 standardize sequencing depth across our dataset, we rarefied (randomly subselected) 2000 
176 sequence reads from each sample. Sequence data have been deposited and made publicly

177 available on Figshare (http://dx.doi.org/10.6084/m9.figshare.1396464).

178 Analyses were conducted in R v. 2.13.1 (R Core Team, 2013), using the vegan package

179 for multivariate analyses (Oksanen et al., 2013) and ggplot2 (Wickham, 2009) to produce plots.

180 We quantified differences in microbial community structure between samples using the Bray-

181 Curtis dissimilarity metric, after square-root transformation. Associations between microbiota

182 structure and species or storage factors were tested with permutational multivariate ANOVAs

183 (Anderson, 2001). The interaction between species and storage variables was included in all such

184 tests. For multivariate analyses, all $\mathrm{R}^{2}$ and $P$ values presented below were calculated using

185 PERMANOVA, and a 0.05 significance threshold was used. Nonparametric Mann-Whitney tests

186 were used to assess differences in the relative abundances of particular taxa between sample

187 treatments and controls with analyses focused on those genera that had median relative

188 abundances $\geq 1 \%$ in at least one sample type, applying a false discovery rate correction to

189 account for multiple comparisons.

190

191 Results and Discussion

192

193 Storage effects

194 The four insect species we examined harbored distinct bacterial communities (Table S1),

195 and the differences in community structure between insect species were far larger than any

196 differences between storage conditions within individual insect species. In a model including

197 insect species, storage treatment, and the interaction between the two, the effect of species was

198 very strong $\left(\mathrm{R}^{2}=0.866, P=0.001\right)$, as is evident from an unconstrained ordination of all the data 
199 where the samples cluster according to insect species they belonged to, and not to the method

200 used to store them (Fig. 2). Overall, we could not reject the null hypothesis of no difference in

201 bacterial community structure among the storage treatments $\left(\mathrm{R}^{2}=0.009, P=0.058\right)$. However,

202 there was a significant interaction, indicating that the strength of a storage effect depended upon

203 the insect species in question $\left(\mathrm{R}^{2}=0.035, P=0.001\right)$.

204 When each insect species was analyzed separately, each of the four insect species

205 exhibited statistically significant effects of storage method on community structure $\left(\mathrm{R}^{2}=0.286-\right.$

$2060.357, \mathrm{P}=0.001-0.019)$, although the magnitude and direction of the effect varied between

207 them (Fig. S1). Thus, storage methods can have effects on insect-associated microbial

208 community structure, but these effects vary by the insect, and are much smaller than species

209 differences. In other words, storing the insect samples in six different ways, including leaving

210 some in empty vials at room temperature for two months, did not hinder our ability to detect

211 species effects, which are often of biological interest (e.g., Colman, Toolson \& Takacs-Vesbach,

212 2012; Jones, Sanchez \& Fierer, 2013; Yun et al., 2014). Our results parallel previous studies on

213 plant, soil, and human fecal microbiota, which did not find strong effects of commonly used

214 storage methods (Lauber et al., 2010; U'Ren et al., 2014; Dominianni et al., 2014; Franzosa et

215 al., 2014). At least for the storage methods tested here, researchers that want to compare bacterial

216 communities across distinct insect taxa using samples stored under different conditions - e.g.,

217 with sample sets compiled from multiple collectors, or in meta-analyses - may not need to

218 worry, at least when the insect species harbor distinct bacterial communities. However, as our

219 focal insects belong to different taxonomic orders with distinct ecological and physiological

220 attributes, specimen storage may be of more concern when investigators are studying more 
221 closely related species. Future research into potential storage biases on microbiota of congeneric

222 insects, for example, would be useful in guiding research at narrower taxonomic scales.

224 bacterial community composition within each of the four insect species examined, we

225 recommend that, when possible, the same method be used to store all specimens within a study.

226 In order to help guide researchers in their choice of storage method, we examined whether some

227 storage methods were consistently better than others, i.e. whether some introduced fewer biases

228 in bacterial community analyses compared to the control. To do so, we tested for storage and

229 species effects on bacterial community structure for each method versus the control. Only the

230 room-temperature unpreserved treatment and DMSO had a (marginally) significant main storage

231 effect, although there was an interaction between species and storage effects in each case (Table

232 1, Fig. S2). Further, the storage effect consistently explained only a small proportion of variation

233 in community structure, especially relative to the species effect (Table 1), which was large and

234 easily visible in ordinations regardless of the storage method used (as mentioned above) (Fig.

235 S2). Ranked by mean Bray-Curtis dissimilarity between each storage method and the control,

236 there was no single 'best' method across all four insect species, and often multiple methods were

237 not substantially distinct from one another in the extent to which the bacterial communities

238 differed from those found in control specimens (Fig. 3). While dependent on the question of

239 interest, we can broadly recommend any of the storage methods tested here, although room-

240 temperature storage without preservative should be avoided when possible.

241 As these analyses were conducted on the structure of the entire bacterial community in

242 each specimen, we also wanted to determine whether the different storage treatments altered the

243 relative abundances of individual, dominant bacterial genera, and whether these effects were 
244 consistent across insect species. We found that genus-level relative abundances were generally

245 not more similar within- than between-treatments, nor markedly different between storage

246 treatments and the control besides natural interindividual variation (Fig. 4). Furthermore, specific

247 genera that differed significantly between a storage method and the control were mostly

248 concentrated in the room temperature treatment, and none differed consistently across insect

249 species (Table S2). This finding suggests that storage conditions are likely to exert their effects

250 by changing the abundance of resident community members, and not by introducing new

251 'contaminant' taxa.

252 Interestingly, although honey bees had the lowest interindividual heterogeneity of the

253 four insects tested (indicated by Fig. 3 and Fig. 4), which should have increased our ability to

254 detect storage-induced effects, they were overall less affected by storage (excepting the room

255 temperature treatment, where Lactobacillus was nearly absent (Fig. 4, Table S2)) than the other

256 insects (Fig. 3). This finding may result from the extremely dense communities inhabiting honey

257 bee guts (possibly reaching ca. $10^{7}$ bacterial colony forming units (CFUs) per bee, (Kwong et al., 258 2014)), which may buffer them from relatively low-abundance contaminants or minor alterations

259 to resident populations. In contrast, bean beetles, where the apparent storage effects were

260 stronger (Fig. 3), have been reported to contain only 600-1750 bacterial CFUs per beetle (Taylor,

261 1985). Bacterial cell counts have not been conducted in A. conspersa and P. rapae adults, but we

262 found substantial interindividual variation in the composition of dominant taxa for all three non-

263 bee species, even within control and treatment groups (Fig. 4). In general, variation in both

264 microbial cell numbers and intra-specific variability in bacterial community composition may

265 explain the observed interactions between storage method and insect species. When feasible,

266 quantifying microbial abundances would be a valuable complement, as storage biases might be 
267 less of a concern for molecular investigations of insect taxa harboring particularly large numbers 268 of microbes.

269

270

271

272

273

274

275

276

277

278

279

280

281

282

283

284

285

286

287

288 289

Surface Sterilization Effects

A second aim of our study was to determine whether surface sterilization affected insectassociated microbiota. As compared with control specimens (which were not surface sterilized), there was no effect of surface sterilization on insect-associated microbial community structure $\left(\mathrm{R}^{2}=0.004, P=0.344\right)$, and no significant interaction between species and sterilization $\left(\mathrm{R}^{2}=\right.$ $0.022, P=0.072)$. Again, samples clustered strongly by species $\left(\mathrm{R}^{2}=0.846, P=0.001\right)$, regardless of whether or not they were surface sterilized (Fig. S3), although there was a slight differentiation between treatments within individual species (excepting honey bees). Likewise, the relative abundances of individual genera were generally similar between sterilized and control specimens (Fig. 4).

While sterilization is usually intended to remove surface contaminants (including those derived from handling the specimen) or microbes typically present on the cuticle, we do not know if these surface-associated microbes are normally abundant enough to be detected in molecular surveys of whole-insect microbiota. In our study, where all insects were collected without gloves, we found that bacteria known to be common on human hands (including Staphylococcus, Corynebacterium, and Propionibacterium; Fierer et al., 2008) were extremely rare or absent from nearly all of our samples. Only Staphylococcus and Corynebacterium were detected and only in control P. rapae insects (at median proportions of 0.0005 and 0.001 , respectively). In light of the general lack of a strong surface sterilization effect at the community level, in tandem with the lack of abundant human-derived contaminants even in control 
290 specimens, we argue that surface sterilization may not be worth the time and effort required.

291 Presumably, the high microbial biomass inside many insects (such as in the gut or

292 bacteriocytes) - relative to microbial colonizers or contaminants on the insect cuticle-

293 overwhelms any potential effect of sterilizing the insect surface prior to microbiota analyses.

294 Although our sterilization protocol was similar to or more aggressive than protocols used in

295 previous studies (e.g., Jones, Sanchez \& Fierer, 2013; Hammer, McMillan \& Fierer, 2014;

296 Sanders et al., 2014), it remains possible that modifications such as a longer soak duration could

297 lead to detectable effects on overall microbiota. However, as we noted earlier, this may have the

298 unintended effect of sterilizing the internal communities that are typically of interest.

299 We recommend omitting surface sterilization from insect microbiota studies, and suggest

300 that any of the storage methods tested here - with the possible exception of room temperature

301 storage without preservative - can be safely used for up to two months if the researcher is not

302 seeking subtle biological patterns that could be obscured by minor storage effects. Each method

303 resolved species differences, and produced reasonably consistent estimates of community

304 structure. Methods may thus be chosen based on practical considerations, such as price,

305 availability, ease of preparation, and travel logistics. For example, CTAB may be a good choice

306 for overseas fieldwork when electricity or dry ice is not available, as it can be maintained at

307 room temperature and is nonflammable. We note, however, that when planning insect collections

308 for microbiota analysis, it is still advisable to standardize methods across all of the specimens.

309 In some cases, researchers are unable to choose how insects are stored in advance, but

310 this should not necessarily limit the utility of those samples for microbial analyses. For example,

311 samples may have already been collected and stored by entomologists for non-microbial

312 purposes (such as morphological analyses). Our data indicate that standard entomological 
313 collection methods, where specimens are not handled aseptically, are sufficient for capturing in

314 situ community structure and biological patterns, and that surface sterilization does not appear to

315 be necessary for microbial studies. Although we do not know how different storage approaches

316 may influence microbial analyses beyond the two month window examined here, we suggest that

317 if storage effects are weak or nonexistent after two months, it is unlikely that they will pose a

318 problem after longer periods of time. However, until longer-term storage effects are explicitly

319 tested, we recommend extracting DNA from specimens within two months of storage when

320 possible.

321 While further investigations into storage effects on insect microbiota would be useful-

322 particularly tests that include combinations of various methods - we anticipate that these

323 findings will allow existing insect samples to be used for microbial DNA sequencing, enable

324 comparative studies that include specimens collected in different ways, and help guide the design

325 and standardization of methods in the rapidly growing field of insect-microbe symbiosis.

326

327 Acknowledgments

328

329

We thank Cesar Nufio, Chelsea Cook, and Megan Blanchard for their assistance

330 collecting insects, and Jessica Henley for her help with DNA sequencing.

331

332 References

333

334 Anderson MJ. 2001. A new method for non-parametric multivariate analysis of variance. Austral 335 Ecology 26:32-46. 
336 Barberán A, Ramirez KS, Leff JW, Bradford MA., Wall DH, Fierer N. 2014. Why are some

337 microbes more ubiquitous than others? Predicting the habitat breadth of soil bacteria.

$338 \quad$ Ecology Letters 17:794-802.

339 Campbell CL, Mummey DL, Schmidtmann ET, Wilson WC. 2004. Culture-independent analysis

340 of midgut microbiota in the arbovirus vector Culicoides sonorensis (Diptera:

341 Ceratopogonidae). Journal of Medical Entomology 41:340-348.

342 Caporaso JG, Lauber CL, Walters WA, Berg-Lyons D, Huntley J, Fierer N, Owens SM, Betley J,

343 Fraser L, Bauer M, Gormley N, Gilbert JA, Smith G, Knight R. 2012. Ultra-high-

344 throughput microbial community analysis on the Illumina HiSeq and MiSeq platforms. The

$345 \quad$ ISME Journal 6:1621-1624.

346 Cary SC, Fierer N. 2014. The importance of sample archiving in microbial ecology. Nature

$347 \quad$ Reviews Microbiology 12:789-790.

348 Colman DR, Toolson EC, Takacs-Vesbach CD. 2012. Do diet and taxonomy influence insect gut 349 bacterial communities? Molecular Ecology 21:5124-5137.

350 Dillon RJ, Dillon VM. 2004. The gut bacteria of insects: nonpathogenic interactions. Annual 351 review of Entomology 49:71-92.

352 Dominianni C, Wu J, Hayes RB, Ahn J. 2014. Comparison of methods for fecal microbiome 353 biospecimen collection. BMC Microbiology 14:103.

354 Douglas AE. 2015. Multiorganismal insects: diversity and function of resident microorganisms. 355 Annual Review of Entomology 60:17-34. 
356 Edgar RC. 2013. UPARSE: highly accurate OTU sequences from microbial amplicon reads. $357 \quad$ Nature Methods 10:996-8.

358 Engel P, Moran NA. 2013. The gut microbiota of insects - diversity in structure and function. 359 FEMS Microbiology Reviews 37:699-735.

360 Estes AM, Hearn DJ, Snell-Rood EC, Feindler M, Feeser K, Abebe T, Dunning Hotopp JC, 361 Moczek AP. 2013. Brood ball-mediated transmission of microbiome members in the dung 362 beetle, Onthophagus taurus (Coleoptera: Scarabaeidae). PLoS ONE 8:e79061.

363 Fierer N, Hamady M, Lauber CL, Knight R. 2008. The influence of sex, handedness, and 364 washing on the diversity of hand surface bacteria. Proceedings of the National Academy of 365 Sciences of the United States of America 105:17994-17999.

366 Franzosa EA, Morgan XC, Segata N, Waldron L, Reyes J, Earl AM, Giannoukos G, Boylan MR, 367 Ciulla D, Gevers D, Izard J, Garrett WS, Chan AT, Huttenhower C. 2014. Relating the 368 metatranscriptome and metagenome of the human gut. Proceedings of the National 369 Academy of Sciences of the United States of America 111:E2329-E2338.

370 Fukatsu T. 1999. Acetone preservation: a practical technique for molecular analysis. Molecular $371 \quad$ Ecology 8:1935-1945.

372 Hammer TJ, McMillan WO, Fierer N. 2014. Metamorphosis of a butterfly-associated bacterial 373 community. PLoS ONE 9:e86995.

374 Jones RT, Sanchez LG, Fierer N. 2013. A cross-taxon analysis of insect-associated bacterial 375 diversity. PLoS ONE 8:e61218. 
376 Koch H, Abrol DP, Li J, Schmid-Hempel P. 2013. Diversity and evolutionary patterns of 377 bacterial gut associates of corbiculate bees. Molecular Ecology 22:2028-2044.

378 Kwong WK, Engel P, Koch H, Moran NA. 2014. Genomics and host specialization of honey bee 379 and bumble bee gut symbionts. Proceedings of the National Academy of Sciences $380 \quad 111: 11509-11514$.

381 Lauber CL, Zhou N, Gordon JI, Knight R, Fierer N. 2010. Effect of storage conditions on the 382 assessment of bacterial community structure in soil and human-associated samples. FEMS 383 Microbiology Letters 307:80-6.

384 Mandrioli M, Borsatti F, Mola L. 2006. Factors affecting DNA preservation from museum385 collected lepidopteran specimens. Entomologia Experimentalis et Applicata 120:239-244.

386 McDonald D, Price MN, Goodrich J, Nawrocki EP, DeSantis TZ, Probst A, Andersen GL, 387 Knight R, Hugenholtz P. 2012. An improved Greengenes taxonomy with explicit ranks for 388 ecological and evolutionary analyses of bacteria and archaea. The ISME Journal 6:610-618.

389 Moreau CS, Wray BD, Czekanski-Moir JE, Rubin BER. 2013. DNA preservation: a test of 390 commonly used preservatives for insects. Invertebrate Systematics 27:81-86.

391 Nagy ZT. 2010. A hands-on overview of tissue preservation methods for molecular genetic 392 analyses. Organisms Diversity \& Evolution 10:91-105.

393 Oksanen J, Blanchet, G, Kindt R, Legendre P, Minchin PR, O'Hara RB, Simpson GL, Solymos

394 P, Stevens MHH, Wagner H. 2011. vegan: Community Ecology Package. Available at 395 http://CRAN.R-project.org/package=vegan 
396 Post RJ, Flook PK, Millest AL. 1993. Methods for the preservation of insects for DNA studies. 397 Biochemical Systematics and Ecology 21:85-92.

398 R Core Team. 2013. R: A language and environment for statistical computing. $R$ Foundation $399 \quad$ Statistical Computing.

400 Ramirez KS, Leff JW, Barberán A, Bates ST, Betley J, Crowther TW, Kelly EF, Oldfield EE, 401 Shaw EA, Steenbock C, Bradford MA, Wall DH, Fierer N. 2014. Biogeographic patterns in 402 below-ground diversity in New York City's Central Park are similar to those observed 403 globally. Proceedings of the Royal Society of London B: Biological Sciences 281:20141988.

404 Rosengaus RB, Zecher CN, Schultheis KF, Brucker RM, Bordenstein SR. 2011. Disruption of 405 the termite gut microbiota and its prolonged consequences for fitness. Applied and $406 \quad$ Environmental Microbiology 77:4303-12.

407 Rubin BER, Gibbons SM, Kennedy S, Hampton-Marcell J, Owens S, Gilbert JA. 2013.

408 Investigating the impact of storage conditions on microbial community composition in soil 409 samples. PLoS ONE 8:e70460.

410 Rubin BER, Sanders JG, Hampton-Marcell J, Owens SM, Gilbert JA, Moreau CS. 2014. DNA 411 extraction protocols cause differences in 16S rRNA amplicon sequencing efficiency but not 412 in community profile composition or structure. MicrobiologyOpen 3:910-921.

413 Sanders JG, Powell S, Kronauer DJC, Vasconcelos HL, Frederickson ME, Pierce NE. 2014.

414 Stability and phylogenetic correlation in gut microbiota: lessons from ants and apes.

$415 \quad$ Molecular Ecology 23:1268-83. 
416 Taylor EC. 1985. Cellulose digestion in a leaf eating insect, the Mexican bean beetle, Epilachna

$417 \quad$ varivestis. Insect Biochemistry 15:315-320.

418 U'Ren JM, Riddle JM, Monacell JT, Carbone I, Miadlikowska J, Arnold AE. 2014. Tissue

419 storage and primer selection influence pyrosequencing-based inferences of diversity and

420 community composition of endolichenic and endophytic fungi. Molecular Ecology

$421 \quad$ Resources 14:1032-1048.

422 Wang Q, Garrity GM, Tiedje JM, Cole JR. 2007. Naive Bayesian classifier for rapid assignment

423 of rRNA sequences into the new bacterial taxonomy. Applied and Environmental

$424 \quad$ Microbiology 73:5261-5267.

425 Wickham H. 2009. ggplot2: elegant graphics for data analysis. Available at

426 http://had.co.nz/ggplot2/book

427 Willows-Munro S, Schoeman MC. 2014. Influence of killing method on Lepidoptera DNA 428 barcode recovery. Molecular Ecology Resources 15:613-618.

429 Yun J-H, Roh SW, Whon TW, Jung M-J, Kim M-S, Park D-S, Yoon C, Nam Y-D, Kim Y-J,

430 Choi J-H, Kim J-Y, Shin N-R, Kim S-H, Lee W-J, Bae J-W. 2014. Insects gut bacterial

431 diversity determined by host environmental habitat, diet, developmental stage and

432 phylogeny. Applied and Environmental Microbiology 80:5254-5264. 


\section{Table $\mathbf{1}$ (on next page)}

Comparison of storage methods

Results from pairwise PERMANOVA tests conducted for samples from each storage method with the controls. Methods are ranked by increasing percentage of variance explained by the storage term. Ordination plots that correspond to these tests are shown in Fig. S2. Significant effects $(P<0.05)$ are indicated by asterisks. 


\begin{tabular}{|l|l|c|c|}
\hline $\begin{array}{l}\text { Storage method } \\
\text { (versus control) }\end{array}$ & Model Term & $\mathbf{R}^{\mathbf{2}}$ & P values \\
\hline Freezer & Species & 0.849 & $0.001^{*}$ \\
\hline & Storage & 0.003 & 0.285 \\
\hline & Species x Storage & 0.047 & $0.002^{*}$ \\
\hline CTAB & Species & 0.862 & $0.001^{*}$ \\
\hline & Storage & 0.005 & 0.149 \\
\hline & Species x Storage & 0.030 & $0.010^{*}$ \\
\hline Ethanol & Species & 0.820 & $0.001^{*}$ \\
\hline & Storage & 0.007 & 0.168 \\
\hline & Species x Storage & 0.035 & $0.016^{*}$ \\
\hline DMSO & Species & 0.839 & $0.001^{*}$ \\
\hline & Storage & 0.011 & $0.040^{*}$ \\
\hline & Species x Storage & 0.046 & $0.001^{*}$ \\
\hline Room Temperature & Species & 0.809 & $0.001^{*}$ \\
\hline & Storage & 0.017 & $0.041^{*}$ \\
\hline & Species x Storage & 0.049 & $0.004^{*}$ \\
\hline
\end{tabular}


Figure $\mathbf{1}_{\text {(on next page) }}$

Experimental design

Overview of how insect samples were collected and processed for later whole-specimen bacterial 16S rRNA gene sequencing. Only beetles are shown, but the design was identical for the other three insect species used in the study. CTAB = cetrimonium bromide, DMSO = dimethyl sulfoxide, $\mathrm{ETOH}=95 \%$ ethanol, $\mathrm{RT}=$ room temperature without preservative, $\mathrm{CTRL}$ $=$ control (no storage or sterilization prior to homogenization), SS = surface sterilized (sterilization but no storage prior to homogenization). 
Host population

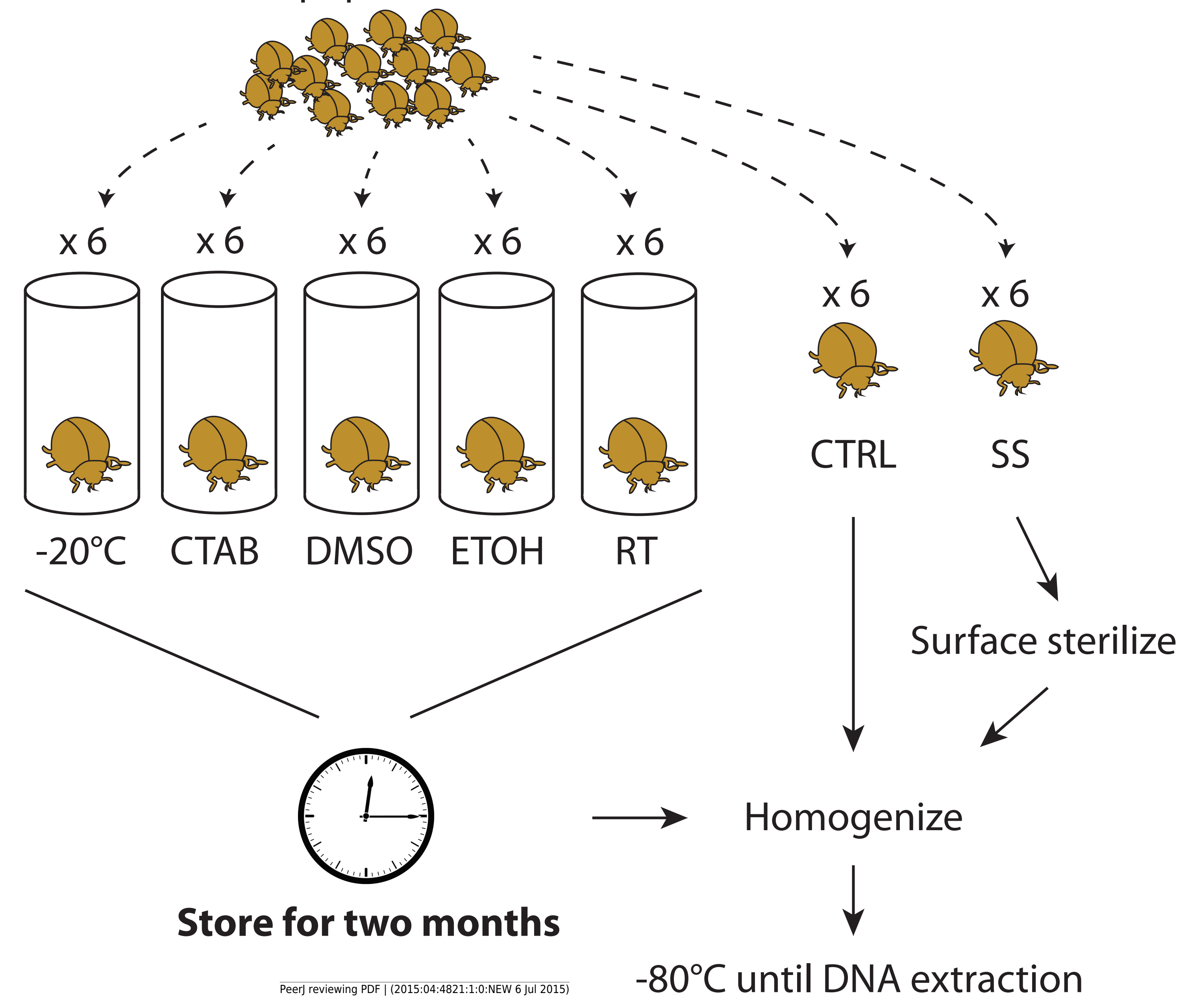


Figure 2 (on next page)

Ordination of insect microbiota

Nonmetric multidimensional scaling ordination showing how insect-associated bacterial communities cluster by host insect species (colors) versus specimen storage method (symbols). Ellipses represent 95\% confidence intervals around species group centroids. 

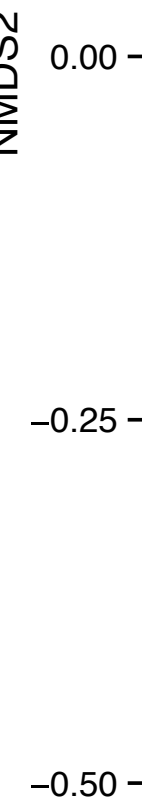

$\triangle$ Control

- CTAB

$\diamond$ DMSO

$\nabla$ Ethanol

$\triangle$ Freezer

Room temp.

Butterfly Grasshopper Bee Beetle 
Figure $\mathbf{3}$ (on next page)

Beta diversity among insect species and storage methods

Boxplots of Bray-Curtis dissimilarities between samples from each storage method and the controls, separated by the four insect species tested. Higher values indicate that the storage method had larger effects on bacterial community structure relative to the control. Note that, due to interindividual variability in the microbiota, there was variation even among the control specimens. CTAB = cetrimonium bromide, DMSO = dimethyl sulfoxide, $\mathrm{ETOH}=95 \%$ ethanol, $\mathrm{RT}=$ room temperature without preservative, $\mathrm{CTRL}=$ control. 
Figure 4 (on next page)

Composition of bacterial communities in study insects

Relative abundances of dominant genera for each individual specimen analyzed in the study. Seventeen genera are displayed, representing the five most abundant from each insect species (three genera were in the top five of more than one insect species). In cases where there was no genus-level identification based on the Greengenes taxonomy, the lowest-level classification is given. Blank areas show the proportion of the community for each sample that does not belong to these dominant genera. CTAB = cetrimonium bromide, DMSO = dimethyl sulfoxide, $\mathrm{ETOH}=95 \%$ ethanol, $\mathrm{RT}=$ room temperature without preservative, $\mathrm{FR}=$ freezer at $-20^{\circ} \mathrm{C}, \mathrm{CTRL}=$ control, $\mathrm{SS}=$ surface-sterilized. 


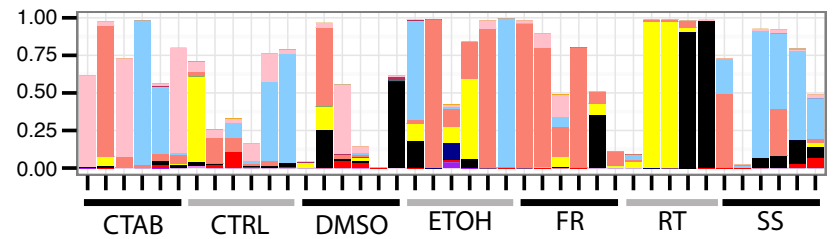

\section{Grasshoppers}

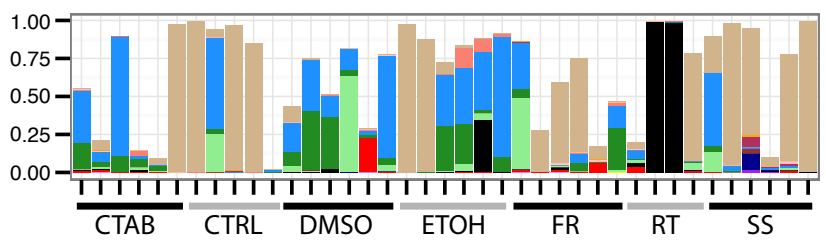

Beetles

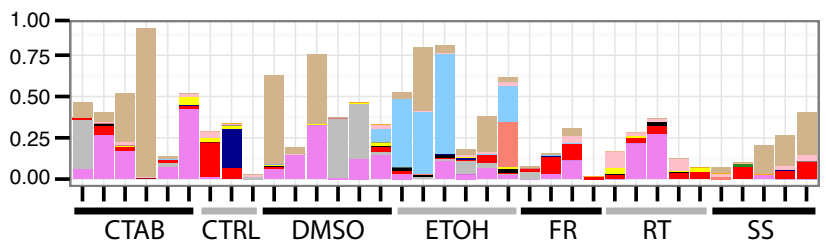

Bees

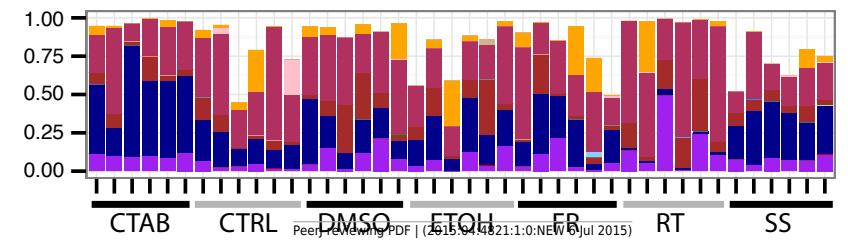

\begin{tabular}{|l}
\hline Rhodococcus \\
Bifidobacterium \\
Phormidiaceae \\
Alicyclobacillus \\
Enterococcus \\
Lactobacillus \\
Lactococcus \\
Neisseriaceae \\
Kingella \\
Neisseria \\
Gammaproteobacteria \\
Klebsiella \\
Providencia \\
Pasteurellales \\
Acinetobacter \\
Pseudomonadaceae \\
Entomoplasmatales \\
\hline
\end{tabular}

